Fortschr. Neurol. Psychiat. 54 (1986) 205-215

(C) Georg Thieme Verlag Stuttgart $\cdot$ New York

\title{
Prognose der kardialen Hirnembolie*
}

\author{
P. Berlit ${ }^{1}$, H. Eckstein ${ }^{1}$, K.-H. Krause ${ }^{2}$ \\ ${ }^{1}$ Neurologische Klinik Mannheim (Ärztlicher Direktor: Prof. Dr. med. O. Hallen) und \\ ${ }^{2}$ Neurologische Klinik Heidelberg (Ärztlicher Direktor: Prof. Dr. med. H. Gänshirt) der Universität Heidelberg
}

\section{Prognosis of cardiogenic cerebral embolism}

In a retrospective study the reports of 211 cases of cardiogenic cerebral embolism - diagnosed on the base of neurological and cardiological findings - were analyzed in view of signs and findings of prognostic value. There were 21 patients with TIA, 39 cases of RIND and 151 patients with cerebral infarction, 60 of which showed mild and 91 severe neurological symptoms. 38 patients died during the period of hospitalization.

While sex of the patients as well as vascular risk factors lhypertension, diabetes mellitus, cigarette smoking) did not influence the clinical course of the disease, patients with TIA or RIND in general were younger (about 5 years) than those with severe stroke.

Prognosis of cardiogenic cerebral embolism depended to a great degree on the underlying heart disease. Cerebral embolism after myocardial infarction showed a better remission of symptoms than embolism in atrial fibrillation. In the group of valvular diseases the course of embolic strokes in mitral lesions was worse than in aortal valve disease. Prognosis was worst in endocarditis, both in view of neurological deficit and of mortality.

Mostly, the cardiogenic emboli lead to infarctions of the middle cerebral artery territory ( 78 per cent) with a predilection for the left hemisphere. In media-syndromes the clinical course was significantly worse in patients with additional homonymous visual defect compared to incomplete in. farctions. Initial disturbance of conscience reduced prognosis quoad vitam et restitutionem significantly.

Of the neuroradiological findings, the detection of arterial occlusion or circulatory disturbance in angiography as well as the finding of an ischemic lesion in computed axial tomography (CAT) was correlated with a severe course of the embolic stroke. While 7 patients with hemorrhagic infarction in CAT-Scan showed no differences in the clinical course, the 14 patients with pathological cerebral spinal fluid findings in embolism had an unfavourable prognosis. The development of epileptic seizures did not influence the further course of the infarction to a significant extent.

Results are compared with the current world literature.

\section{Zusammenfassung}

In einer retrospektiven Studie wurden die Krankengeschichten von 211 Patienten, bei denen aus neurologischer und kardiologischer Sicht die Diagnose einer kardialen Hirnembolie gestellt worden war, ausgewertet im Hinblick auf prognostisch relevante Befunde. Bei 21 Patienten traten transitorisch ischämische Attacken (TIAs), bei 39 Kranken reversible ischämische neurologische Defizite (RINDs) auf; 151 Patienten erlitten einen Hirninfarkt, wobei es bei 91 Kranken zu schweren neurologischen Symptomen und bei 60 nur zu funk tionell wenig beeinträchtigenden neurologischen Ausfällen kam. 38 Patienten verstarben während des stationären Aufenthaltes.

Patienten mit einer transitorischen neurologischen Symptomatik waren durchschnittlich fünf Jahre jünger als jene mit schweren persistierenden neurologischen Symptomen. Das Geschlecht der Kranken und das Vorliegen der Gefäßrisiko- fak toren Hypertonus, Diabetes mellitus und Nikotinabusus hatten keinen Einfluß auf den klinischen Verlauf der Erkrankung.

Die Prognose der kardialen Hirnembolie war wesentlich beeinflußt durch die zugrundeliegende kardiale Emboliequelle. Hirnembolien nach Herzinfarkt zeigen eine bessere Symptomrückbildung als embolische Hirninfark te bei absoluter Arrhythmie bei Vorhofflimmern. Bei den Herzklappenfehlern war die Prognose bei solchen der Mitralklappen schlechter als bei Aortenvitien. Die ungünstigste Prognose sowohl im Hinblick auf Symptomrückbildung als auch auf Mortalität zeigten die entzündlichen Herzerkrankungen.

Am häufigsten war das Versorgungsgebiet der Arteria cerebri media durch die Hirnembolien betroffen $(78 \%)$ mit deutlicher Bevorzugung der linken Hemisphäre. Bei Mediasyndromen war der klinische Verlauf bei Patienten mit zusätzlicher homonymer Hemianopsie signifikant schlechter als bei solchen mit Mediateilinfarkten. Das initiale Auftreten einer Bewußtseinsstörung (Somnolenz bis Koma) führte zu einer signifikanten Verschlechterung der Prognose quoad restitutionem et quoed vitam.

Unter den neuroradiologischen Befunden hatte sowohl der positive angiographische Nachweis einer Gefäßokklusion bzw. einer Zirkulationsstörung als auch der Befund eines Hirninfarktes in der axialen Computertomographie des Schädels eine ungünstige Bedeutung hinsichtlich des weiteren klinischen Verlaufes. 14 Patienten mit pathologischem Liquorbefund zeigten einen ungünstigen Verlauf, 7 Patienten mit computertomographisch nachwe isbarer Hämorrhagie unterschieden sich hinsichtlich der Prognose nicht sicher vom restlichen Kollektiv.

Dem Auftreten von epileptischen Anfällen kam in der vorliegenden Studie keine sichere Bedeutung für die Prognose der kardialen Hirnembolie zu.

Die Ergebnisse werden mit den Angaben der Weltliteratur verglichen.

\section{Einleitung}

Die bei weitem häufigste Ursache des Hirninfarktes sind arteriosklerotische Läsionen vornehmlich der extrakraniellen hirnversorgenden Arterien, welche über einen Gefäßverschluß, über hämodynamische Auswirkungen bei hochgradiger Stenose und durch arterio-arterielle Embolien die Gewebsischämie bedingen. Neben der Embolisierung ausgehend von arteriosklerotischen Plaques vorgeschalteter Gefäße, kann auch das Herz Ausgangspunkt für zerebrale Embolien sein. Die Häufigkeitsangaben im Schrifttum zur

*Ein Teil der Ergebnisse der vorliegenden Arbeit wurde auf dem 13. Weltkongreß der Neurologie in Hamburg, 1. bis 6.9.1985, vorgetragen. 
kardialen Hirnembolie schwanken beträchtlich. In prospektiven Untersuchungen liegen die Zahlenangaben zwischen 13 und $23 \%$ (Garraway et al. 1979, Mohr et al. 1978), in retrospektiven klinischen Übersichten werden Prozentzahlen zwischen 9 und 45,7\% genannt (Gautier u. Morelot 1977, Gottstein 1977). In Autopsiestudien liegen die Zahlenangaben durchweg höher als in klinischen Arbeiten (Blackwood et al. 1969, Jörgensen u. Torvik 1969, Sturgill u. Netsky 1963, Vost et al. 1964, Zang $X u$ 1979).

Die hohe Schwankungsbreite der Zahlenangaben, vornehmlich im klinischen Schrifttum, dürfte ihre Ursache in erster Linie in der Problematik einer adäquaten Diagnostik zum Nachweis einer kardialen Hirnembolie haben. Sichere klinische Unterscheilungsmerkmale zwischen thrombotischem und kardial-embolischem Hirninfarkt gibt es nicht, obwohl eine Reihe von Charakteristika die Verdachtsdiagnose einer kardialen Hirnembolie am Krankenbett stellen lassen. Hierzu zählen vor allem das plötzliche Auftreten von schweren neurologischen Ausfällen, die einem - oft linkshirnigen Media(teil)infarkt entsprechen, in den Vormittagsstunden bei körperlicher Betätigung sich manifestieren und mit Kopfschmerzen und Bewußtseinsstörung einhergehen (Berlit u. Eckstein 1985). Weitere wichtige Verdachtsmomente für die Annahme der kardial-embolischen Genese eines Hirninfarktes sind ein eher jüngeres Alter des Patienten und das Fehlen der klassischen Gefäßrisikofaktoren. Die angiographische Abklärung im Rahmen der neuroradiologischen Diagnostik des Hirninfarktes kann einen wesentlichen Beitrag zur Diagnosestellung liefern. Wird angiographisch eine Läsion der extrakraniellen hirnversorgenden Gefäße ausgeschlossen und finden sich Gefäßokklusionen in der Peripherie, so stellt diese Befundkonstellation ein wesentliches Argument für die Annahme einer kardialen Genese dar. Der Nachweis eines Embolus in situ an einer arteriosklerose-atypischen Stelle kann ebenso wie ein unauffälliger Befund in der Reangiographie bei zuvor nachgewiesener Okklusion oder hochgradiger Stenosierung die embolische Genese eines Hirninfarktes beweisen (Berlit et al. 1983, Bladin 1964). Jedoch können bei unauffälligem extrakraniellen Befund an den Karotiden der Nachweis eines Mediahauptstammverschlusses, eines Mediaastverschlusses oder einer Zirkulationsstörung im Versorgungsgebiet der Arteria cerebri media ebenfalls als typisch für eine Hirnembolie gelten (Berlit 1983). Beim Bestehen klinisch schwerer neurologischer Ausfälle wird ein vollkommen regelrechter angiographischer Gefaßbefund eher für als gegen die Annahme einer kardialen Hirnembolie sprechen. $\mathrm{Ob}$ angiographisch der Nachweis eines embolietypischen Gefaßßbefundes gelingt oder nicht, hängt in erster Linie vom Zeitpunkt der angiographischen Untersuchung ab; je früher die Kontrastmitteluntersuchung durchge führt wird, desto eher ist mit dem Nachweis eines Strombahnhindernisses zu rechnen (Berlit 1983).

Die Prognose der kardialen Hirnembolie wird von einer Reihe von Faktoren bestimmt: Es müssen neben dem Schweregrad der neurologischen Ausfallerscheinungen insbesondere die zugrunde liegende Herzerkrankung, die Ausgangspunkt des Embolus gewesen ist, und die Verhältnisse am Hirngefäßsystem selbst Beruicksichtigung finden. Anders als beim thrombotischen Hirninfarkt hat der Hirnkreislauf bei der kardialen Hirnembolie nicht die Gelegenheit, im Laufe der Progredienz eines arteriosklerotischen Plaque einen mehr oder weniger suffizienten Kollateralkreislauf auszubilden. Schließlich dürften neurologische Begleitsymptome und internistische Begleiterkrankungen neben dem Alter der Patienten e ne Rolle spielen.

Die letzten ausführlichen Ubersichten über die Prognose der Hirnembolie haben Carter 1965 und Wells 1959 gegeben. In der vorliegenden Arbeit soll versucht werden, anhand der retrospektiven Auswertung von 211 Verlaufsbeobachtungen bei kardialer Hirnembolie Kritierien furr die Beurteilung der Prognose des Krankheitsbildes herauszuarbeilen.

\section{Material und Methodik}

In der vorliegenden Studie wurden die Krankengeschichten von 211 Patienten mit einer Hirnembolie kardialer Genese im Hinblick auf Symptome, neuroradiologische und kardiologische Befunde sowie Verlauf ausgewertet. Alle Kranken hatten sich wegen eines Hirninfarktes in stationärer neurologischer Behandlung befunden, in jedem Falle war aus neurologischer Sicht die Verdachtsdiagnose einer Hirnembolie geäußert worden und war von kardiologischer Seite eine Herzerkrankung als mögliche kardiale Emboliequelle gefunden worden. Patienten, bei denen zwar aufgrund klinischer oder neuroradiologischer Befunde die Verdachtsdiagnose einer kardialen Hirnembolie gestellt worden war, der Kardiologe jedoch keine in Frage kommende Emboliequelle nachweisen konnte, fanden in der vorliegenden Arbeit keine Berücksichtigung.

Um Aussagen über die prognostische Bedeutung einze ner Befunde machen zu können, wurden die $211 \mathrm{Krankenge-}$ schichten anhand der neurologischen Symptomatik und des klinischen Verlaufes in funf Schweregrade eingeteilt:

Gruppe 1: Transitorisch ischämische Attacke (TIA) mit vollständiger Rückbildung der neurologischen Symptome innerhalb von 24 Stunden.

Gruppe 2: Reversibles ischämisches neurologisches Defizit (RIND); neurologische Symptomatik vollständig reversibel innerhalb von maximal 3 Wochen.

Gruppe 3: Hirninfarkte (a) mit nur geringen funktionellen Residualsymptomen, die den Patienten nicht in seiner Selbständigkeit beeinträchtigen.

Gruppe 4: Hirninfarkte (b) mit deutlichen Residualsy:mptomen; die Patienten sind mit Unterstützung gehfähig, zeigen aber eine schwere Halbseitensymptomatik.

Gruppe 5: Hirninfarkte (c) ohne Rückbildung der neurologischen Symptomatik. Die Patienten sind pflegeabhängig, häufig bettlägerig oder an den Folgen des Hirninfarkte's verstorben.

Die statistische Auswertung erfolgte mittels des U-Testes von Wilcoxon, Mann und Whitney.

\section{Ergebnisse}

Bei den 211 Patienten mit einer kardialen Hirnembolie handelte es sich um 102 Frauen und um 109 Männer. Bei einem Durchschnittsalter von 60,5 Jahren des Gesamtkrankengutes zeigte das weibliche Geschlecht mit 63,7 Jahren ei ᄀ höheres Durchschnittsalter als die männlichen Patienten mit 


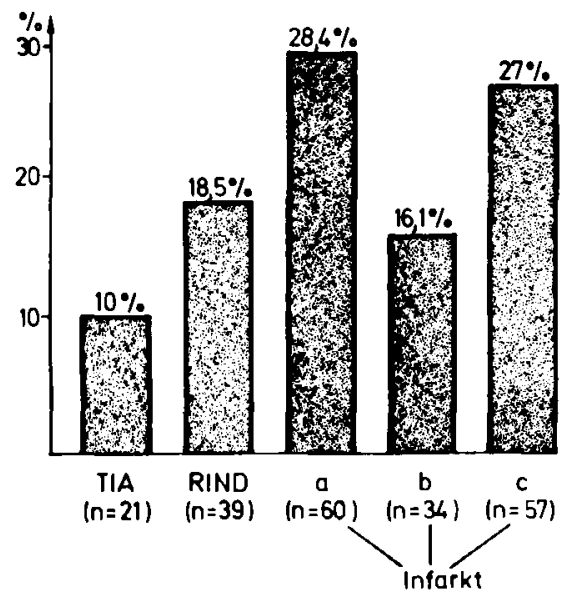

Abb. 1 Klinischer Verlauf bei 211 Patienten mit kardialer Hirnembolie

57,5 Jahren. Durchschnittsalter und Geschlecht wurden in großem Umfange von der Alters- und Geschlechtsverteilung der zugrundeliegenden kardialen Emboliequelle bestimmt: Während bei der Patientengruppe mit Herzinfarkt, Kardiomyopathie und entzündlichen Herzerkrankungen das männliche Geschlecht überwog, waren Frauen häufiger von Hirnembolien bei Herzrhythmusstörungen, Herzvitien und dem Mitralklappenprolaps betroffen. Mit einem Durchschnittsalter von $67 \mathrm{~J}$ ahren waren die Kranken mit einer Herzrhythmusstörung im Durchschnitt signifikant älter als jene mit einem Herzinfarkt (Durchschnittsalter 62 Jahre), Herzvitien (Durchschnittsalter 56 Jahre), Kardiomyopathien (Durchschnittsalter 52 Jahre) und entzünd lichen Herzerkrankungen (Durchschnittsalter 50 Jahre).

Als kardiale Emboliequellen wurden in der Reihenfolge ihrer Häufigkeit folgende Herzerkrankungen gefunden: in $46 \%(n=97)$ lagen Herzrhythmusstörungen vor, wobei es sich bei 68 Kranken um eine absolute Arrhythmie bei Vorhofflimmern handelte; in 12 Fällen lag ein intermittierendes Vorhofflimmern, 9 mal das Syndrom des kranken Sinusknotens vor. Seltene Ursachen für Herzrhythmusstörungen waren eine Thyreotoxikose und eine Digitalisintoxikation. Bei 53 Patienten trat die Hirnembolie bei Herzklappenfehler auf, wobei es sich um 37 Mitralvitien (davon 13 kombiniert) und 10 Aortenvitien (6 kombiniert) sowie 2 Vorhofseptumdefekte handelte. Bei 30 Patienten lag zusätzlich eine Herzrhythmusstörung vor. Nach einem Herzinfarkt ereignete sich die Hirnembolie bei 25 Patienten, wobei die Herzvorderwand einschließlich des Septums am häufigsten betroffen war. Bei 11 Kranken lag eine Kardiomyopathie als Grunderkrankung vor, wobei es sich bei 8 Patienten um eine dilatative Kardiomyopathie handelte; in der Mehrzahl der Fälle bestand der Verdacht auf eine alkoholtoxische Kardiomyopathie. Unter zehn Fällen einer entzündlichen Herzerkrankung handelte es sich $7 \mathrm{mal}$ um eine Endokarditis und 3 mal um eine Myokarditis. In fünf Fällen wurde ein Mitralklappenprolaps als mögliche Emboliequelle nachgewiesen, zusätzliche kardiale Erkrankungen lagen in keinem Fall vor. Eine paradoxe Embolie bei offenem Foramen ovale wurde ebenfalls bei fünf Kranken angenommen. Bei drei Pa-

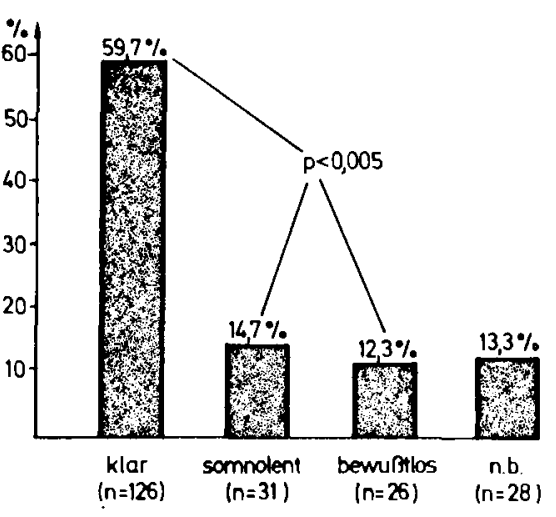

Abb. 2 Initiale Bewußtseinslage bei 211 Patienten mit kardialer Hirnembolie (n.b. = nicht bekannt)

tienten schließlich trat die Hirnembolie bei künstlicher Herzklappe auf.

Die klinische Symptomatik war bei 168 Patienten durch ein Mediasyndrom bestimmt, wobei $117 \mathrm{mal}$ die Arteria cerebri media links und 51 mal die mittlere Hirnarterie rechts betroffen war. In knapp $40 \%$ handelte es sich klinisch um einen Mediateilinfarkt, in $45 \%$ bestand neben dem Vollbild eines Mediasyndroms auch eine homonyme Hemianopsie zur Gegenseite. Während die vordere Hirnarterie nur bei zwei Patienten durch den embolischen Verschluß betroffen war, fand sich eine Symptomatik von seiten der Arteriae cerebri posteriores bei 24 Kranken. Bei weiteren 23 Patienten war das vertebro-basiläre Stromgebiet betroffen, wobei es sich zumeist um Hirnstamminfarkte im Sinne eines mehr oder weniger ausgeprägten WallenbergSyndroms und um Kleinhirninfarkte handelte. Aber auch lakunäre Infarkte mit umschıriebenen Ausfällen im Bereichı von Hirnnerven und Blickmotorik wurden beobachtet.

Aufgrund des klinischen Verlaufes wurde die Einteilung der Patienten in die eingangs genannten füf Gruppen vorgenommen, die Ergebnisse sind in Abb. 1 zusammengestellt. Es zeigt sich, daß bei $28,5 \%$ der Patienten mit kardialer Hirnembolie eine transitorische neurologische Symptomatik vorlag, bei weiteren $28,4 \%$ führte der resultierende Hirninfarkt nur zu funktionell wenig beeinträchtigenden neurologischen Ausfällen. Bei einem Drittel der Kranken dagegen resultierte ein ausgedehnter Hirninfarkt, der den Patienten auf Dauer von der Hilfe Dritter abhängig machte.

38 Patienten ( $18 \%$ ) verstarben nach stattgehabter kardialer Hirnembolie während des stationären Aufenthaltes. Bei 6 Patienten fuihrte eine obere oder untere Einklemmung als direkte Folge des embolischen Hirninfarktes zum Tode, wobei die Uberlebenszeit in dieser Patientengruppe im Median 1,5 Tage betrug. Bei weiteren 6 Kranken kam es im Verlauf der ersten drei Wochen nach dem Hirninfarkt zu einem zentral bedingten Herzkreislaufversagen. Insgesamt war bei diesen Patienten, die an den direkten Folgen des Hirninfarktes verstarben, $9 \mathrm{mal}$ die Arteria cerebri media und $3 \mathrm{mal}$ die $\mathrm{Ar}$ teria basilaris betroffen. Bei 7 Kranken führte (im Median 


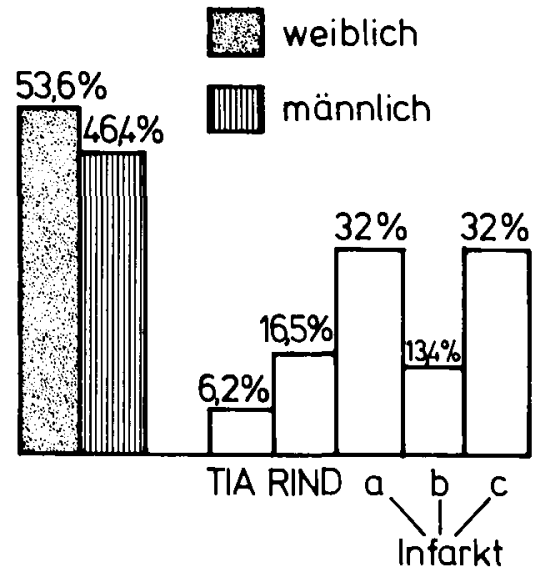

Herzrhythmusstörungen (n=97)

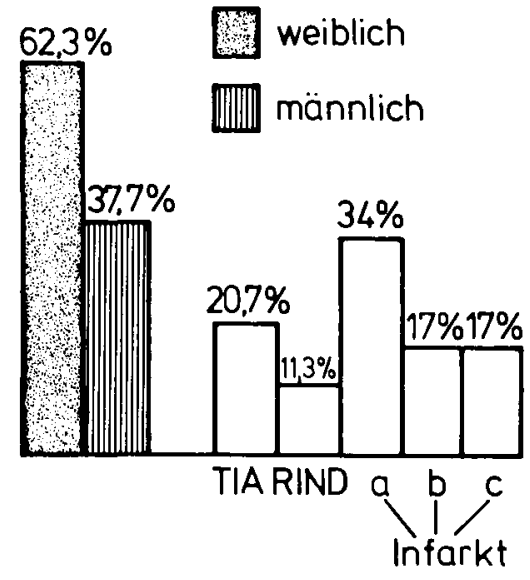

Herzfehler $(n=53)$
Abb. 3a

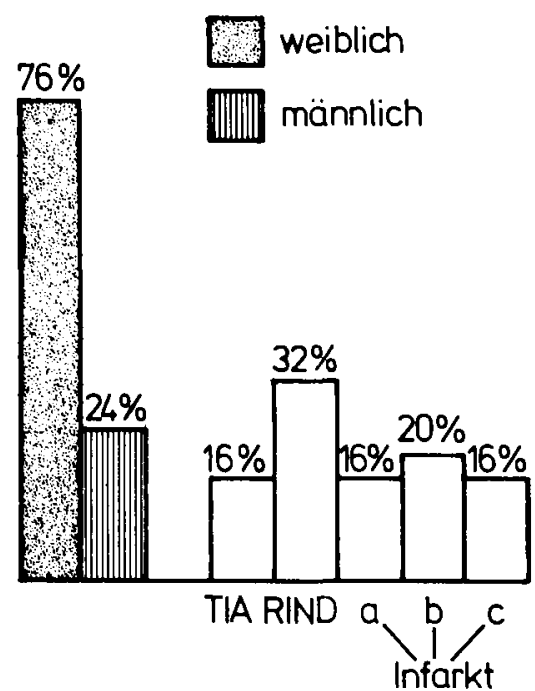

Herzinfarkt $(n=25)$
Abb. 3b

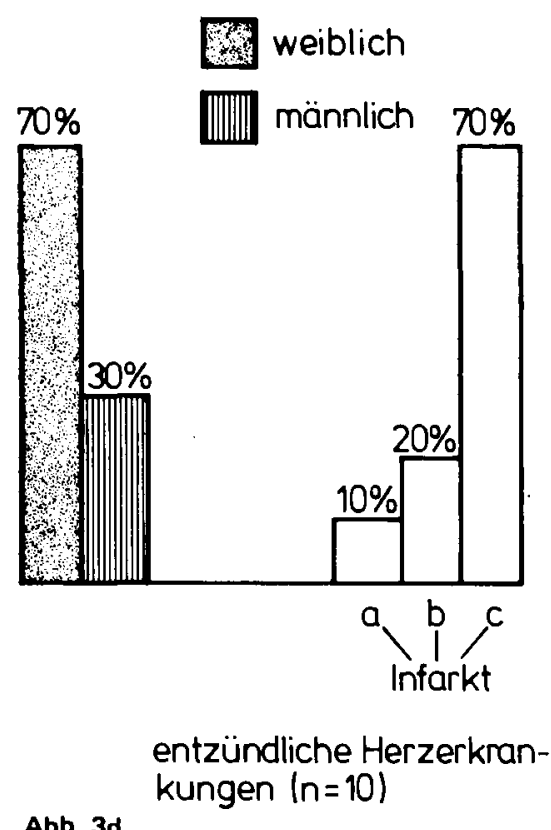

Abb. 3d

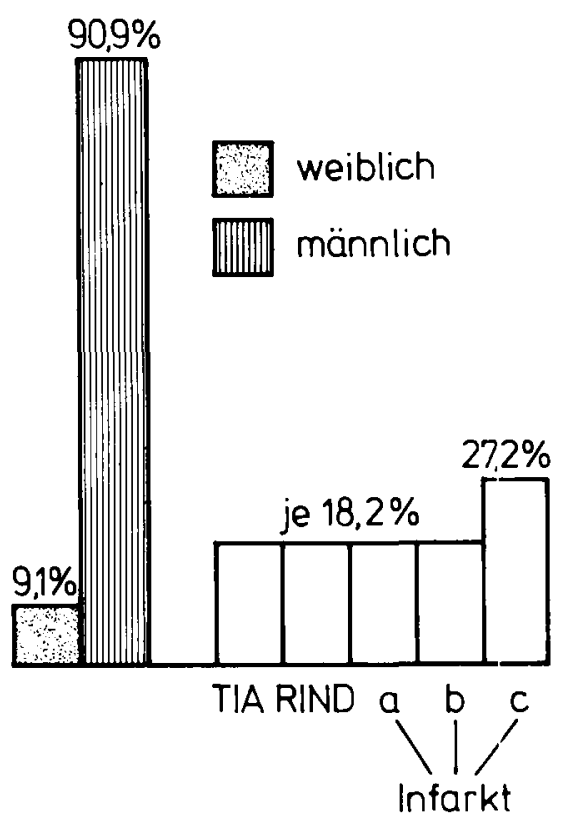

Kardiomyopathie (n:=11)

Abb. 3c

Abb. 3 a-e Geschlechtsverteilung und klinischer Verlauf in Abhängigkeit von der zugrundeliegenden Herzerkrankung

nach 22 Tagen) eine erneute Embolie ad exitum. Hierbei handelte es sich $5 \mathrm{mal}$ um Lungenembolien, einmal um einen embolischen Hirnstamminfarkt und einmal um eine Mesenterialarterienembolie. 15 Patienten starben an den Folgen der zugrundeliegenden kardialen Erkrankung, wobei es sich $4 \mathrm{mal}$ um einen Reinfarkt des Herzens handelte. Bei $2 \mathrm{~Pa}$ tienten führte eine Pneumonie, bei einer Kranken eine Hirnblutung unter Antikoagulantientherapie zum Tode.

Begleitsymptome waren bei den 211 Patienten mit kardialer Hirnembolie unseres Krankengutes Kopfschmerzen bei 30 Patienten (14,2\%), symptomatische epileptische Anfälle bei 22 Kranken $(10,4 \%)$ und initiale Bewußtseinsstörungen bei 57 Patienten ( $27 \%$ ). Während den Kopfschmerzen und dem Vorhandensein von epileptischen Anfällen keine prognostische Bedeutung im Hinblick auf Restitution und Mor- talität zukommt, verschlechtert eine initiale Bewußtseinsstörung die Prognose quoad restitutionem und quoad vitam signifikant (Abb. 2). Während unter 57 Patienten mit initialer Somnolenz oder Bewußtlosigkeit lediglich 9 Fälle mit transitorischer'neurologischer Symptomatik auftraten, lagen TIAs und RINDs bei einem Viertel der 126 Patienten ohne Bewußtseinsstörung vor $(\mathrm{p}<0,005)$.

Ein weiterer klinischer Befund, der eine prognostische Aussage bei den Patienten zuließ, war das Vorhandensein einer homonymen Hemianopsie bei einem Mediasyndrom. Beim statistischen Vergleich von Patienten mit der Symptomatik eines Mediateilinfarktes (häufig Aphasie mit diskreter Monoparese des rechten Armes) und solchen mit einer komp etten Mediasymptomatik, einschließlich homonymem Gesichtsfeldausfall, ergab eine hochsignifikant bessere Rückbildung 

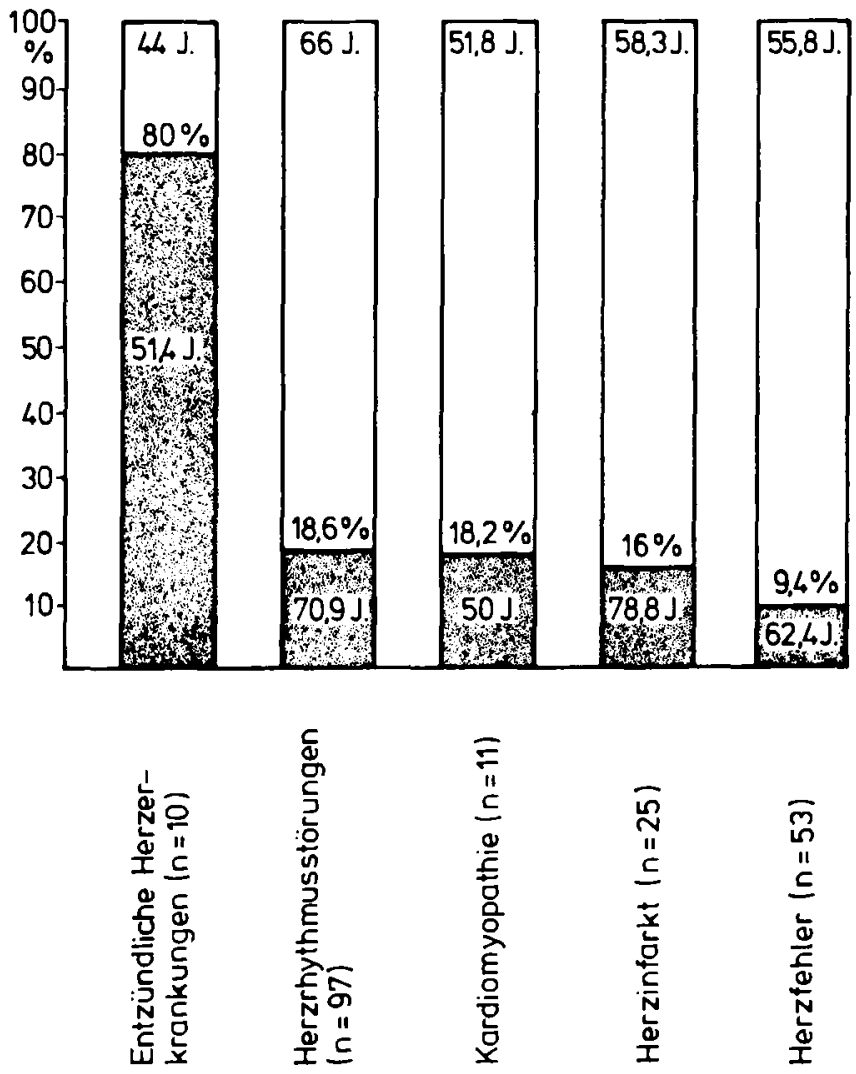

Abb. 4 Mortalität der Hirnembolie bei verschiedenen kardialen Emboliequellen. - verstorbene Patienten, $\square$ überlebende Patienten. Es ist das Durchschnittsalter in Jahren für die einzelnen Untergruppen in den Säulen angegeben

der Symptome bei den Teilinfarkten $(p<0,01)$. Linke und rechte Hemisphäre unterschieden sich hinsichtlich der Rückbildungsraten ebenso wenig wie Hirninfarkte im vorderen bzw. hinteren Hirnkreislauf. Zehn Kranke boten initial eine Deviation conjugée; aus dieser Gruppe verstarben drei Patienten, drei weitere blieben bettlägerig, fielen somit ebenfalls in die Gruppe c. Insgesamt ist die Zahl von zehn Patienten jedoch für eine statistische Aussage zu gering.

Für die Prognose der karäialen Hirnembolie spielte die zugrundeliegende Herzerkrankung in unserem Krankengut eine entscheidende Rolle. In Abb. 3 ist der klinische Verlauf bei den einzelnen Herzerkrankungen und in Abb. 4 die Mortalität bei den einzelnen Herzerkrankungen zusammengestellt. Bei den Herzrhythmusstörungen, der häufigsten kardialen Emboliequelle, kam es bei fast jedem zweiten Patienten zu schweren irreversiblen neurologischen Symptomen. Eine transitorische Symptomatik lag bei $23 \%$ dieser 97 Patienten vor. 18 Kranke (19\%) mit Herzrhythmusstörungen verstarben während des stationären Aufenthaltes, bei einem Viertel der Patienten kam es zu dauernder Pflegebedürftigkeit durch den embolischen Hirninfarkt. Das Durchschnittsalter der verstorbenen Kranken lag mit 71 Jahren fünf Jahre über dem Alter derer, die die Hirnembolie überlebten.

Bei immerihin 23 (32\%) der 53 Patienten mit einem Herzvitium kam es zu einer TIA bzw. einem RIND. Weitere $34 \%$ zeigten geringe funktionell wenig beeinträchtigende bleibende neurologische Symptome (a), so daß lediglich bei einem Drittel der Kranken mit Herzfehler schwere bleibende neurologische Ausfälle resultierten. Fünf Patienten kamen in dieser Gruppe ad exitum; der Altersunterschied zwischen Uberlebenden (55,8 Jahre) und Verstorbenen $(62,4$ Jahre) betrug etwa 6 Jahre. Die Prognose in der Gruppe der Herzvitien hing entscheidend mit davon ab, ob eine zusätzliche Herzrhythmusstörung vorlag. Bei 17 Patienten mit einem Mitralfehler, die zusätzlich ein Vorhofflimmern aufwiesen, war der klinische Verlauf hinsichtlich Remission und bleibenden Ausfällen deutlich ungünstiger als bei den 10 Patienten mit Mitralfehler und erhaltenem Sinusrhythmus ( $p<0,05)$. Während in der größeren Gruppe der Mitralvitien das Vorliegen einer isolierten Insuffizienz oder Stenose bzw. einer Kombination beider Anteile im Hinblick auf die Prognose keine Bedeutung hatte, unterschieden sich die Kranken mit einem Mitralvitium von denen mit einem Aortenvitium. Durchweg war der funktionelle Status bei Entlassung in der Gruppe der Mitralvitien schlechter, wobei dies am ehesten auf das häufigere gleichzeitige Vorliegen einer Herzrhythmusstörung in dieser Gruppe zurückzuführen war.

In der Gruppe der Patienten mit einer Hirnembolie nach Herzinfarkt $(n=25)$ lagen transitorische Symptome in fast der Hälfte der Fälle vor; auch hier fanden sich schwere bleibende neurologische Ausfallerscheinungen bei gut einem Drittel der Kranken.

Unter den vier verstorbenen Patienten führte bei einer Kranken die zwei Wochen nach Auftreten des embolischen Hirninfarktes eingeleitete Antikoagulantientherapie zu einer tödlichen Massenblutung. Insgesamt war der Altersunterschied zwischen verstorbenen und überlebenden Patienten mit über 20 Jahren in der Gruppe der Herzinfarktpatienten besonders eindrucksvoll.

Eine besonders schlechte Prognose sowohl im Hinblick auf die Symptomrückbildung als auch im Hinblick auf die Mortalität hatten die entzündlichen Herzerkrankungen, TIAs und RINDs wurden bei Endo- oder Myokarditis in keinem Fall beobachtet, lediglich einer der zehn Kranken wies nur geringgradige neurologische Symptome auf. Acht Kranke verstarben während des stationären Aufenthaltes, wobei diese durchschnittlich älter waren als jene zwei, die überlebten.

In der Gruppe der 11 Patienten mit einer Kardiomyopathie waren symptomatische epileptische Anfalle bei 27,3 \% vertreten. Dies ist wesentlich mehr als der Prozentsatz von $10,4 \%$ für das Gesamtkollektiv. Diese Beobachtung findet ihre Erklärung vermutlich darin, daß bei mehr als der Hälfte der Patienten mit Kardiomyopathie eine alkoholtoxische Genese anzunehmen war. In über der Hälfte der embolischen Ereignisse war die Prognose quoad restitutionem günstig. Zwei Patienten verstarben während des stationären Aufenthaltes, wobei diese beiden interessanterweise etwas jünger waren als jene, die überlebten.

Bei den selteneren Emboliequellen zeigten die Hirnembolien bei Mitralklappenprolapssyndrom und bei künstlicher Herzklappe im allgemeinen eine gute Rückbildungstendenz, während die paradoxen Hirnembolien bei offenem Foramen ovale in der Regel in schweren neurologischen bleibenden Ausfällen resultierten. 


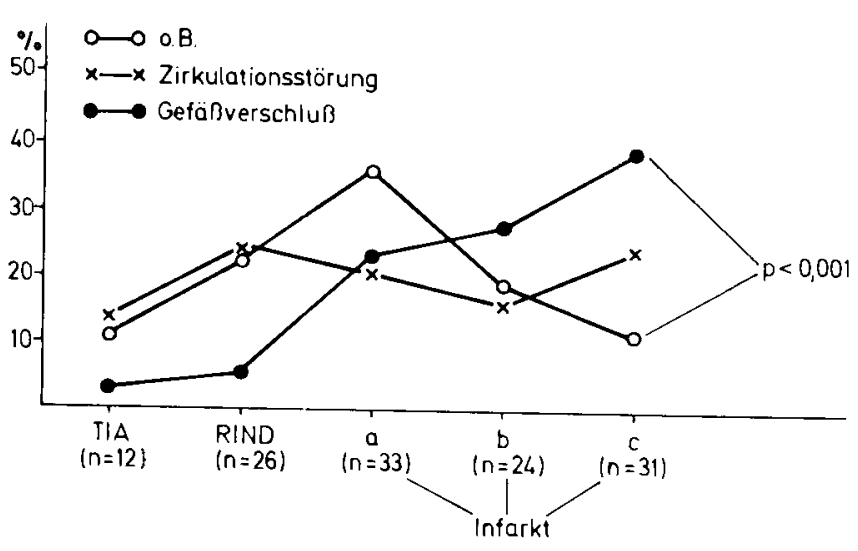

Abb. 5 Klinischer Verlauf und angiographischer Befund bei 126 Patienten mit kardialer Hirnembolie

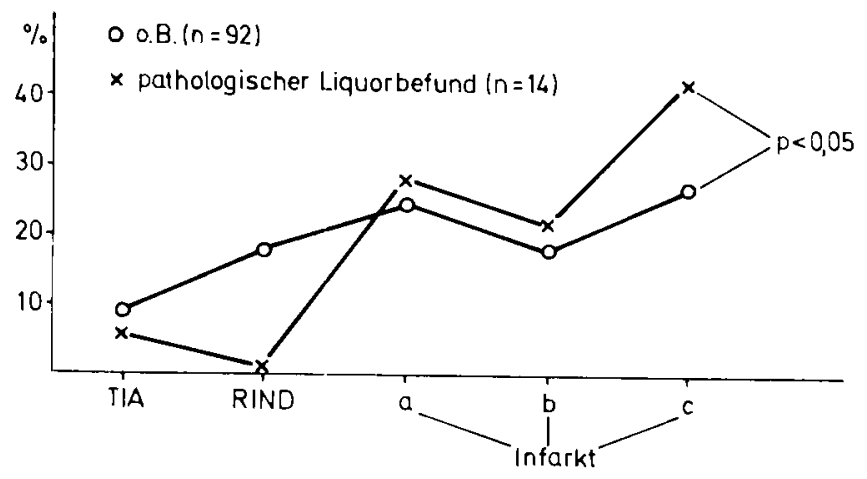

Abb. 7 Klinischer Verlauf und Liquorbefund bei 106 Patienten mit kardialer Hirnembolie

Zusammenfassend ist die Prognose der Hirnembolie in der Gruppe der Herzinfarktpatienten und der Herzvitien am günstigsten, in jener der entzündlichen Herzerkrankungen besonders ungünstig einzuschätzen. Vor allem die Mortalität ist bei den Karditiden mit $80 \%$ gegenüber einem Gesamtdurchschnitt von $18 \%$ signifikant gegenüber den anderen Herzerkrankungen erhöht $(p<0,01)$. Aber auch die größte Gruppe der Embolien bej Herzrhythmusstörungen hat hinsichtlich der Rückbildung der neurologischen Symptomatik eine eher ungünstige Prognose.

\section{Zusatzuntersuchungen}

Eine angiographische Untersuchung der hirnversorgenden Gefaße erfolgte bei 126 Patienten. Die hierbei erhobenen Befunde wurden bereits anderenorts ausführlich dargestellt (Berlit 1983, Berlit u. Eckstein 1985). Die am häufigsten erhobenen Befunde waren ein vollkommen unauffalliger Gefaßßstatus ( $n=44)$, ein Gefäßverschluß, wobei zumeist die Arteria cerebri media bzw. einer ihrer Äste betroffen war $(n=38)$ sowie Zirkulationsstörungen ebenfalls vornehmlich im Media-Territorium $(n=29)$. Vergleicht man den klinischen Verlauf der angiographisch untersuchten Patienten, so zeigt sich, daß jene mit einem angiographisch faßbaren Befund häufiger schwere bleibende neurologische Aus-

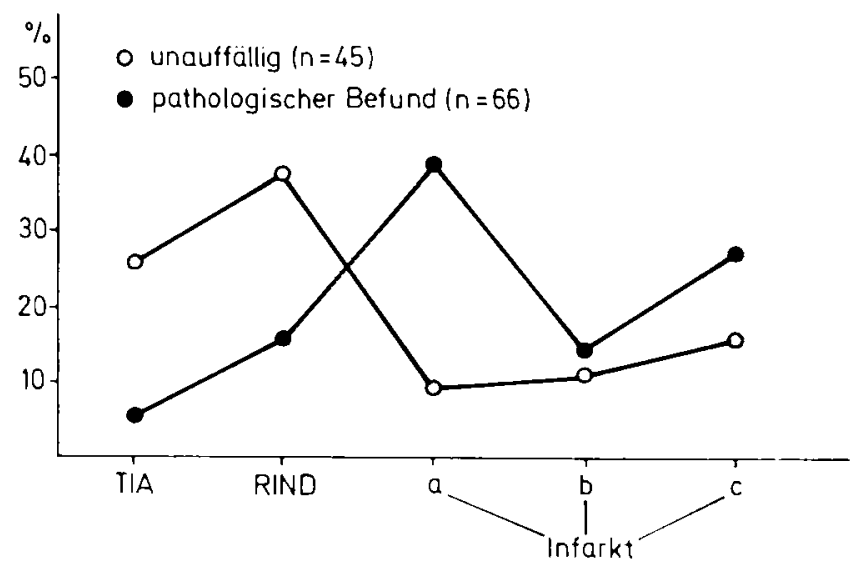

Abb. 6 Klinischer Verlauf der kardialen Hirnembolie in Abhängigkeit vom computertomographischen Befund bei 111 Patienten

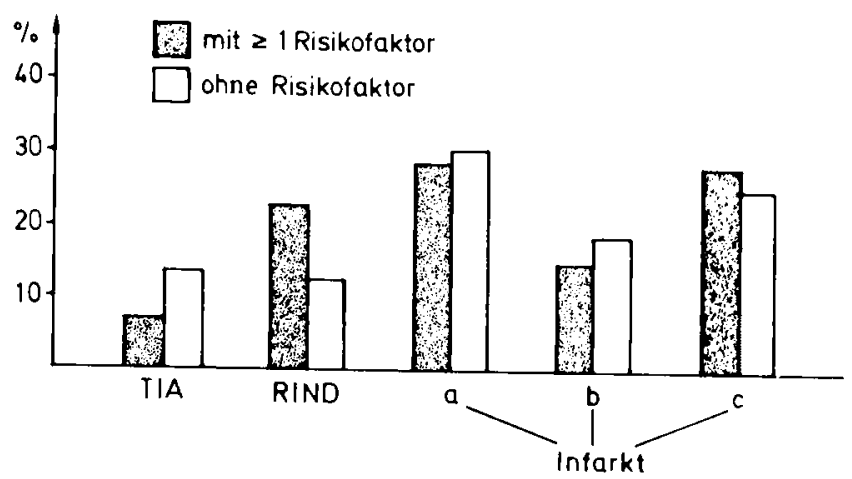

Abb. 8 Gefäßrisikofak toren (Hypertonus, Diabetes mellitus, Nikotinabusus) und klinischer Verlauf bei 211 Patienten mit kardialer Hirnembolie

fälle zeigen, wobei in der Gruppe c der Unterschied zwischen unauffälligem angiographischen Befund und nachgewiesener Gefäßokklusion signifikant ist $(p<0,001$, Abb. 5).

Auch ein nachweisbarer Infarktbezirk in der axialen $\mathrm{Ccm}_{\mathrm{Cm}}$ putertomographie des Schädels sprach in der Regel für Jas Vorliegen eines Hirninfarktes mit mehr oder weniger funktionell beeinträchtigenden bleibenden Ausfällen; jedoch kamen auch bei einzelnen TIA-bzw. RIND-Patienten hypodense Läsionen zur Darstellung, und es gab Kranke, bei denen trotz unauffälligem Computertomogramm eine teleibende Halbseitenlähmung bestand (Abb. 6). Sieben der insgesamt 111 computertomographisch untersuchten Patienten zeigten Einblutungen in den Infarktbezirk; diese unters:hieden sich nicht sicher von den restlichen Patienten im Hinblick auf klinischen Verlauf und Mortalität.

Eine Lumbalpunktion erfolgte bei 106 Kranken nach Auftreten des embolischen Hirninfarktes. Hierbei wurde in 14 Fällen ein pathologischer Befund erhoben, wobei es sich oft um einen fleischwasserfarbenen Liquor mit leichter Pleozy. tose handelte, seltener auch um Eiweißerhöhungen. Die neurologischen Ausfallserscheinungen bildeten sich bei pathologischem Liquorbefund in der Regel nicht vollstän- 


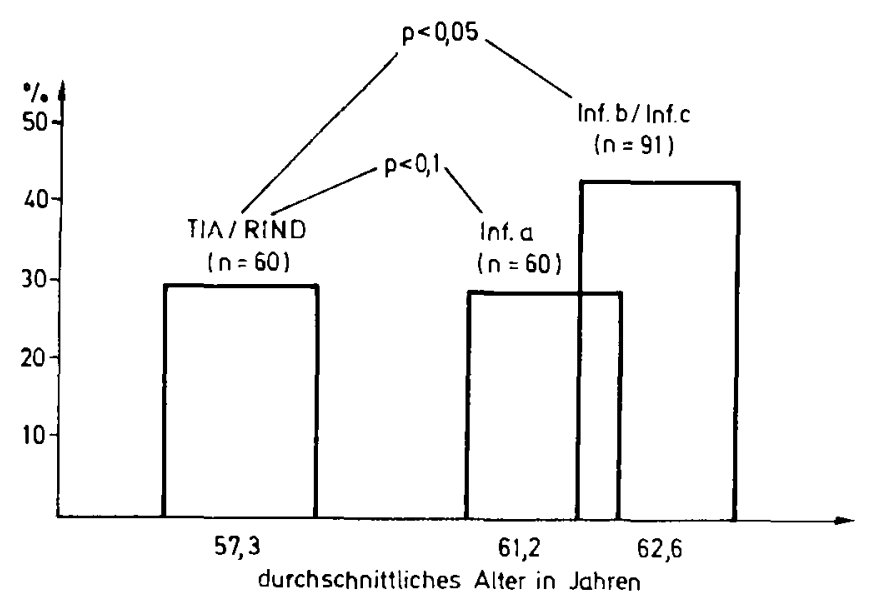

Abb. 9 Lebensalter und klinischer Verlauf der kardialen Hirnembolie $(n=211)$

dig zurück; im Hinblick auf schwere bleibende neurologische Symptome zeigte sich ein signifikanter Unterschied zur Gruppe jener Patienten mit unauffalligem Liquor ( $p<0,05$, Abb. 7).

Die Gefäßrisikofaktoren Hypertonus, Diabetes mellitus und Nikotinabusus spielten hinsichtlich der Prognose keine sichere Rolle. Wie sich aus Abb. 8 ergibt, lassen sich keine verwertbaren Unterschiede ableiten. Insgesamt waren die genannten Risikof aktoren nur in einem relativ niedrigen Prozentsatz vertreten: eine arterielle Hypertonie lag bei $39 \%$ der $\mathrm{Pa}$ tienten, ein Diabetes mellitus bei $18 \%$ und ein Nikotinabusus bei $17 \%$ vor. Weitere Parameter, welche erfaßt wurden, aber keine Rolle im Hinblick auf die Prognose spielten, waren die Hyperurikämie (bei 5,2\%), die Einnahme von Ovulationshemmern $(2,8 \%)$ und das Vorliegen einer Hyperlipidämie (bei 9,5\%).

Läßt man die verschiedenen kardialen Emboliequellen unberücksichtigt, so zeigt sich für das Gesa mtkollektiv von 211 Patienten ein signifikanter Unterschied des Lebensalters für Kranke mit transitorischer Symptomatik im Vergleich zu jenen mit irreversiblen schweren neurologischen Ausfällen bzw. Exitus letalis (Abb. 9); Patienten mit TIA oder RIND waren im Durchschnitt 5 Jahre jünger.

\section{Diskussion}

Seit den Arbeiten von Wells 1959 und Carter 1965 gilt die Prognose der kardialen Hirnembolie generell als ungünstig im Vergleich zum thrombotischen Hirninfarkt. Daß vornehmlich die Mortalität bei der kardialen Hirnembolie höher ist, bestätigten 1980 Easton u. Sherman, die über eine Mortalität von ca. $30 \%$ berichteten, und 1983 Caplan et al. in der Michael Reese Stroke Registry. In der prospektiven Framinghamstudie hingegen, in der 5184 Menschen über 26 Jahre im Hinblick auf das Auftreten von Hirninfarkten verfolgt wurden, war die Dreißig-Tage-Mortalität bei den embolischen Infarkten mit $16 \%$ nicht sicher höher als bei den thrombotischen (15\%) (Sacco et al. 1982). Eine Reihe von Autoren betont, daß das Lebensalter der Patienten zum Zeit- punkt des Auftretens der kardialen Hirnembolie im Hinblick auf die Mortalität eine entscheidende Rolle spielt (Baker et al. 1968, Balow et al. 1966, Coulshed et al. 1970, Wells 1959). Dementsprechend ist die Langzeitprognose bei Arbeiten, die sich ausschließlich mit Patienten mit einem Alter von unter 40 Jahren beschäftigen, günstiger ( $A$ uff et al. 1984, Grindal et al. 1978). In unserer Krankengruppe von 211 Patienten mit kardialer Hirnembolie betrug das Durchschnittsalter $60,5 \mathrm{~J}$ ahre, wobei die Altersklasse unter 40 Jahren bei 7,6\% vertreten war. Das Durchschnittsalter unseres Kollektives liegt damit unter den 68,4 Jahren (Caplan et al. 1983) bzw. 75 Jahren (Jörgensen u. Torvik 1966) vergleichbarer klinischer bzw. pathologischer Studien. Die Ursache hierfür dürfte in erster Linie sein, daß die genannten Autoren in ihren Arbeiten lediglich komplette Hirninfarkte, nicht jedoch TIAs und RINDs auswerteten. Die 60 Patienten unseres Kollektivs, welche eine transitorische Symptomatik boten, waren mit 57,3 Lebensjahren signifikant jünger als jene 91 Kranken, die einen kompletten Hirninfarkt mit schweren bleibenden neurologischen Ausfällen erlitten bzw. an ihrer Erkrankung verstarben (Durchschnittsalter 62,6 Jahre). Geschlechtsunterschiede im Hinblick auf die Prognose sahen wir bei unseren Patienten nicht, auch im Schrifttum finden sich entsprechende Angaben nicht. Hingegen gibt es zur Bedeutung der Gefäßrisikofaktoren, insbesondere des erhöhten Blutdruckes, im Schrifttum unterschiedliche Angaben: Während McAllen u. Marshall 1977 und Mohr u. Mitarb. 1978 eine schlechtere Langzeitprognose bei Patienten mit einer Hypertonie berichteten, fanden Hassan et al. 1978 keinen sicheren Einfluß von Gefaßrisikofaktoren. Auch wir konnten eine ungünstigere Prognose bei dem im Vergleich zum thrombotischen Hirninfarkt niedrigen Prozentsatz an Patienten mit den Gefaßrisikofaktoren arterielle Hypertonie, Diabetes mellitus, Hyperlipidämie und Nikotinabusus nicht beobachten.

Eine entscheidende Bedeutung im Hinblick auf die Prognose der kardialen Hirnembolie kam in der vorliegenden Studie den zugrundeliegenden Herzerkrankungen zu. Betrachtet man die häufigsten Emboliequellen, so war die Langzeitprognose der Hirnembolie bei Patienten nach Myokardinfarkt und mit Klappenfehlern günstiger als bei der größten Gruppe der Herzrhythmusstörungen; besonders hoch war die Mortalität bei den entzündlichen Herzerkrankungen. Herzrhy thmusstörungen, insbesondere die absolute Arrhythmie bei Vorhofflimmern, wird heute von vielen Autoren - auch ohne das Vorliegen von Herzvitien oder sonstigen Herzerkrankungen - als Ausgangspunkt für systemische Embolien anerkannt (Aberg 1969, Barolin 1981, Beer u. Ghitman 1961, Kuramoto et al. 1984, Millikan 1983). Eine besondere Emboliegefährdung wird beim Wechsel bradykarder und tachykarder Rhythmusstörungen (Fairfax et al. 1976) und bei Rhythmusstörungen im Rahmen einer Thyreotoxikose (Forfar et al. 1977) berichtet. In unserem Krankengut machten die Herzrhythmusstörungen mit 97 Patienten fast die Hälfte aller kardialen Emboliequellen aus. Transitorische Symptome lagen bei $23 \%$ der $97 \mathrm{Kran}-$ ken dieser Gruppe vor; dies entspricht den Befunden von Gautier u. Morelot 1977, während Caplan et al. 1983 und Mohr et al. 1978 mit $11 \%$ eine niedrigere Rate berichten. Insgesamt ist die Prognose mit einer Mortalität von $19 \%$ und schweren persistierenden neurologischen Ausfällen in 
$25 \%$ bei Hirnembolien im Rahmen einer Herzrhythmusstörung eher ungünstig einzuschätzen. Die Häufigkeit von Rezidivembolien wurde prospektiv mit $14 \%$ von Hart $\mathrm{u}$. Mitarb. 1983 ermittelt.

Die besonders schlechte Prognose bei entzündlichen Herzerkrankungen mit einer Letalität von $80 \%$ und schweren persistierenden neurologischen Ausfällen bei den restlichen Kranken in unserem Kollektiv wurde auch von anderen Autoren beobachtet. Carter berichtete 1965 sogar über eine Mortalität von $100 \%$ bei seinen Patienten. Harrison u. Hampton 1967 gaben $67 \%$ an. Ursächlich spielten weniger die Größe der entstehenden Hirninfarkte - die Embolien bei Endokarditis sind häufig kleiner als zum Beispiel bei Klappenfehlern (Gahl u. Schliack 1980) -, als vielmehr die Komplikationen durch das entzündliche Geschehen, insbesondere die Ausbildung mykotischer Aneurysmata eine Rolle (Lichtlen 1985).

Bei den 53 Patienten unseres Krankengutes mit einem Herzvitium war die Prognose in über $65 \%$ sowohl quoad vitam als auch quoad restitutionem günstig. Die Mortalität lag mit 9,4 \% deutlich unter den von Gahl u. Schliack 1980 genannten $30 \%$. Die Embolieinzidenz bei Mitralvitien wird mit et wa $20 \%$ beziffert (Lillicrap u. Piesowicz 1964), sie liegt damit höher als die entsprechenden Angaben für Aortenvitien. Interessanterweise treten allerdings nach Klappenersatz Embolien häufiger bei künstlichen Aortenklappen auf (Friedli et al. 1971). Die Prognose wird beim gleichzeitigen Bestehen einer Herzrhythmusstörung vornehmlich bei Mitralstenosen deutlich schlechter (Carter 1965, Coulshed et al. 1970, Daley et al. 1951 und Dewar u. Weightman 1983). Auch wir beobachteten einen signifikanten Unterschied hinsichtlich Remission neurologischer Ausfälle beim Vergleich von Mitralfehlern mit und ohne Herzrhythmusstörung.

Während im Patientenkollektiv von Carter (1965) die Prognose bei Embolien nach Herzinfarkt schlechter als bei solchen nach Klappenfenlern und Herzrhythmusstörung war, hatten diese Patienten in unserer Krankengruppe eine relativ günstige Prognose. Bleibende neurologische Ausfallserscheinungen bestanden bei einem Drittel der Kranken, lediglich vier Patienten verstarben. Im Hinblick auf die Mortalität kam in der Gruppe der Herzinfarktpatienten bei uns dem Alter die größte Bedeutung zu mit einem Altersunterschied von über 20 Jahren $z w i s c h e n$ verstorbenen und überlebenden Patienten. Eine entsprechende Beobachtung teilten McAllen u. Marshall 1977 mit.

Die elf Patienten mit einer Hirnembolie bei Kardiomyopathie zeigten überwiegend eine gute Symptomrückbildung ihrer neurologischen Ausfalle, boten allerdings in einem hohen Prozentsatz (27\%) symptomatische epileptische Anfälle. Eine wesentliche Rolle dürfte bei dieser Beobachtung die Tatsache gespielt haben, daß in über der Hälfte dieser Kranken eine alkoholtoxische Genese der Kardiomyopathie anzuneinmen war (Berlit u. Krause 1981). Bei der idiopathischen Form der Kardiomyopathie zeigen die sich im jüngeren Alter manifestierenden Erkrankungen oft einen schlechteren Verlauf als jene, die sich erst später manifestieren (Delius et al. 1976, Kuhn et al. 1982); dies könnte erklären, warum auch in unserem Krankengut die zwei Patienten, die verstarben, jünger als das Restkollektiv waren. Insgesamt sollen Hirnembolien bei der Kardiomyopathie eher selten auftreten, an neurologischen Symptomen werden häufiger Synkopen beobachtet; eine bevorzugte Embolielokalisation scheinen die Mesenterialgefäße zu sein (Cosnett u. Pudifin 1964, Demakis et al. 1974, Kuhn et al. 1982).

Bei den selteneren Emboliequellen unseres Kollektivs bestätigen sich im wesentlichen die Erfahrungen anderer Autoren hinsichtlich der Prognose. So zeigen die neurologischen Symptome bei Mitralklappenprolapssyndrom eine meist gute Rückbildung (Barnett et al. 1976 und 1980, Fieschi et al. 1983), Folgen einer paradoxen Embolisierung bei offenem Foramen ovale hatten eine durchweg ungünstigere Prognose quoad restitutionem (Berlit et al. 1983, Jones et al. 1983).

Symptomatische epileptische Anfalle verschlechterten in der Übersichtsarbeit von Wells 1959 die Prognose kardialer Hirnembolien. Die Angaben über die Inzidenz epileptischer Anfälle im Schrifttum schwanken erheblich: währer d Mohr et al. 1978 lediglich bei $4 \%$ von 215 Hirnembolien epileptische Anfälle beobachteten, berichteten Furlan et al. 1982 über eine Inzidenz von $42 \%$ Epilepsien. Die von uns beobachtete Häufigkeit von 10,4\% entspricht im wesent ichen den entsprechenden Angaben auch beim thrombotischen Infarkt (Cocito et al. 1982), wir konnten keinen sicheren Einfluß auf die Prognose der Hirnembolie nachweisen.

Auf die ungünstige prognostische Bedeutung einer Bewußtseinsstörung beim Hirninfarkt unterschiedlicher Genese wiesen Sindermann u. Kuntze 1971 hin: von 27 bew ußtseinsgestörten Schlaganfallpatienten verstarben in ihrem Krankengut 18, die häufigste Todesursache war eine Pneumonie. Grindal u. Mitarb. (1978) beobachteten den Verlauf bei 58 Schlaganfallpatienten im Alter bis zu 40 Jahren, vei denen es sich in der Hälfte der Fälle um Hirnembolien handelte. Während bei $3 / 4$ ihrer Kranken eine gute Prognose zu verzeichnen war, verstarben 8 Patienten, dies entsprich1 $14 \%$; 7 dieser 8 Patienten waren initial bewußtseinsgestör gewesen. Auf die ungünstige Prognose bei Hirnemboliepatienten mit Bewußtseinsstörung hat bereits Wells 1959 hingewiesen. In unserem Krankengut kamen 57 Patienten mit initialer Somnolenz oder Bewußtlosigkeit zur Beobachtung; in der Regel handelte es sich um ausgedehnte Hirninfarkte mit schweren persistierenden neurologischen Ausfällen, die den Kranken auf Dauer auf die Hilfe Dritter anwiesen. Auch die 6 Patienten mit einer overen oder unteren Einklemmung als Todesursache fielen durchweg in diese Gruppe. Das initiale Auftreten einer Deviation conjugée bei der Hirnembolie zeigte eine Tendenz zu schlechterer Rückbildung der neurologischen Ausfälle im Verlauf bei 10 Patienten, die wir beobachteten; dies entspricht der Mitteilung von Kayser-Gatchalian 1983.

Das klinisch-neurologische Bild der Hirnembolie erla ubte bei der größten Gruppe der Mediainfarkte insofern e ne Aussage über die weitere Prognose, als das Hinzutreten einer homonymen Hemianopsie beim Mediasyndrom die Prognose signifikant verschlechterte. Die - insgesamt etwas selteneren - Mediateilinfarkte, welche als embolietypisch gelten (van Horn u. Howes 1982), zeigten eine bessere Rückbildungstendenz. Uber die von Balow et al. 1966 mitgeteilte Beobachtung einer ungünstigeren Prognose bei 
embolischen Hirninfarkten unter Miteinbeziehung des Anteriorversorgungsgebietes können wir aufgrund unserer $\mathrm{Be}$ funde keine Aussage machen, da lediglich zwei Patienten einen Anteriorinfarkt aufwiesen. Allerdings zeigten diese beiden Kranken nur eine schlechte Remission der neurologischen Symptome.

Unter den apparativen Verfahren kommt der angiographischen Darstellung der hirnversorgenden Gefäße für die Diagnose einer kardialen Hirnembolie die größte Bedeutung zu (Berlit 1983, Zatuchni et al. 1961, Zang Xu 1979), wobei die Chance eines positiven angiographischen Befundes mit zunehmendem Abstand zwischen Hirninfarkt und Angiographie abnimmt (Alter et al. 1972). In ihrer Autopsiestudie wiesen Torvik u. Jörgensen 1966 darauf hin, daß die Prognose bei Hirnembolien vornehmlich dann ungünstiger war, wenn bei der Sektion eine Gefäßokklusion nachweisbar war. Dies entspricht der von uns gemachten Beobachtung, daß diejenigen Kranken mit einem angiographisch faßbaren Befund häufiger schwere bleibende neurologische Ausfälle zeigten, wobei die Prognose um so ungünstiger war, je später die Gefäßokklusion noch nachgewiesen werden konnte.

Auf die Häufigkeit hämorrhagischer Infarkte bei Hirnembolien haben Fisher u. Adams 1950 hingewiesen, wobei sie dieses Phänomen vornehmlich durch sekundäre Einblutungen nach Rekanalisation eines zuvor verschlossenen Gefäßes erklärten. Nach größeren autoptischen Studien bestehen Hämorrhagien in embolischen Infarkten in über $60 \%$ der Fälle (Jörgensen u. Torvik 1969). Diese scheinen jedoch in einem hohen Prozentsatz dem Auflösungsvermögen der Computertomographie als diagnostischer Methode der Wahl zu entgehen (Berlit 1983). Weisberg berichtet in seiner computertomographischen Studie von 35 Hirnembolien über das Bild eines hämorrhagischen Infarktes bei 7 Kranken (Weisberg 1985), in unserem Patientengut waren bei 111 entsprechend untersuchten Patienten lediglich 7 Hämorrhagien nachweisbar. Während der positive Nachweis eines Infarktes - vor allem mit den Zeichen der Raumforderung sich ungünstig auf die Prognose auswirkte, spielte der Nachweis einer Hämorrhagie für den weiteren Verlauf keine sichere Rolle.

106 Patienten unseres Krankenkollektivs wurden nach Auftreten der kardialen Hirnembolie lumbal punktiert, ein pathologischer Befund wurde bei 14 Kranken erhoben. Diese zeigten eine signifikant schlechtere Rückbildung ihrer neurologischen Ausfälle. Entsprechende Beobachtungen werden im Schrifttum nicht mitgeteilt.

Die Prognose der kardialen Hirnembolie wird neben der Gefährdung durch den aufgetretenen Hirninfarkt und die zugrundeliegende Herzerkrankung wesentlich durch die Gefahr von Rezidivembolien bestimmt. Während Hakim et al. 1983 in ihrer prospektiven Studie nur bei $10 \%$ Embolie. rezidive beobachteten, berichten Koller 1982 über $25 \%$, Wells 1959 über $30 \%$ und Carter 1965 bei einer Beobachtungszeit zwischen 6 und 12 Jahren sogar über bis zu $75 \%$ Rezidivembolien. Der Großteil der erneuten Embolien tritt innerhalb der ersten zwölf Monate nach dem initialen Ereignis auf, Furlan et al. 1982 beobachteten $13 \%$ Embolierezidive sogar innerhalb der ersten sieben Tage nach embolischem Hirninfarkt. Easton u. Sherman 1980 geben an, daß sich ein Drittel aller Rezidivembolien im ersten Monat ereignet. Wir sahen bei unseren 211 Kranken 38 Embolierezidive, wobei sich $40 \%$ innerhalb der ersten drei Wochen nach Herzinfarkt ereigneten. In sieben Fällen führte - im Median nach 22 Tagen - die Rezidivembolie zum Tode. Hierbei waren häufiger andere Organe als das Gehirn durch die erneute Embolie betroffen. Vor allem diese Beobachtungen von Rezidivembolien, vornehmlich in den ersten drei bis vier Wochen nach Hirnembolie, führten im Schrifttum in zunehmendem Maße zur Empfehlung einer frühzeitigen Antikoagulation, wenn zuvor computertomographisch ein hämorrhagischer oder deutlich raumfordernder Infarkt ausgeschlossen wurde (Furlan et al. 1982, Fleming u. Bailey 1971, Hakim et al. 1983). Calandre et al. 1984 empfehlen den Beginn der Antikoagulation ab dem 4. Tag nach Auftreten der Hirnembolie, weil es in den ersten drei Tagen noch häufiger zu sekundären Hämorrhagien kommen soll. Grundsätzlich besteht die Gefahr der Umwandlung einer weißen in eine rote Malazie bei früher Antikoagulantienbehandlung, und die Diskussion über Risiko und Nutzen der frühen Antikoagulantienbehandlung bei der kardialen Hirnembolie ist noch nicht abgeschlossen.

\section{Literatur}

Aberg, $H .:$ A trial fibrillation: 1. A study of atrial thrombosis and systemic embolism in necropsy material. Acta Med. Scand 185 (1969) 373-379

Alter, M., S. Kieffer, J. Resch, K. Ansari: Cerebral infarction. Clinical and angiographic correlations. Neurology 22 (1972) $590-602$

Auff, E., G. Schnaberth, K. Zeiler: Langzeitprognose von Patienten mit juvenilem Insult, katamnestische Ergebnisse. Eur. Arch. Psychiatr. Neurol. Sci. 234 (1984) 275-280

Baker, R.N., W.S. Schwartz, J.C. Ramseyer: Prognosis among survivors of ischemic stroke. Neurology 18 (I968) 933-941

Balow, J., M. Alter, J. Resch: Cerebral thromboembolism. Neurology 16 (1966) 559-564

Barnett, H.J.M., M.W. Jones, D.R. Boughner, W.J. Kostuk: Cerebral ischemic events associated with prolapsing mitral valve. Arch. Neurol. 33 (1976) 777-782

Barnett, H.J.M., D.R. Boughner, D.W. Taylor, P.E. Cooper, W.J. Kostuk, P.M. Nichol: Further evidence relating mitral-valve prolapse to cerebral ischemic events. N. Engl. J. Med. 302 (1980) 139-144

Barolin, G.S.: Zur kardialen (Mit-) Verursachung von Hirninfarkt und TIA. In: Lang, E. (Hrsg.): Kardiale Ursachen zerebrovaskulärer Syndrome. Springer-Verlag Berlin, Heidelberg, New York (1981) 93-107

Beer, D.T., B. Ghitman: Embolization from the atria in ateriosclerotic heart disease. JAMA 177 (1961) 287-291

Berlit, $P$.: Die kardiale Hirnembolie. Nervenarzt 54 (1983) 389399

Berlit, P., H. Eckstein: Diagnose der kardialen Hirnembolie aus neurologischer Sicht. In: Gänshirt, H., P. Berlit, G. Haack (Hrsg.). Kardiovaskuläre Erkrankungen und Nervensystem. Springer Verlag Berlin, Heidelberg, New York, Tokyo 1985

Berlit, P., K.H. Krause: Die Hirnembolie bei der kongestiven Kardiomyopathie des Alkoholikers. Nervenarzt 52 (1981) 605-607

Berlit, P., P. Vetter, K.H. Krause: Die paradoxe Hirnembolie. Nervenarzt 54 (1983) 311-315

Blackwood, W., J.F. Hallpike, R.S. Kocen, W.G.P. Mair: Atheromatous disease of the carotid arterial system and embolism from the heart in cerebral infarction: a morbid anatomical study. Brain 92 (1969) 897-910

Bladin, P.F.: A radiological and pathologic study of embolism of the internal carotid-middle cerebral artery axis. Radiology 82 (1964) 615-624 
Calandre, L., J.F. Ortega, F. Bermejo: Anticoagulation and hemorrhagic infarction in cerebral embolism secondary to rheumatic heart disease. Arch. Neurol. 41 (1984) 1152-1154

Caplan, L.R., D.B. Hier, J. D'Cruz: Cerebral embolism in the Michael Reese Stroke Registry. Stroke, 14 (1983) 530-536

Carter, A.B.: Prognosis of cerebral embolism. Lancet 2 (1965) 514 519

Cocito, L., E. Farale, L. Reni: Epileptic seizures in cerebral artery occlusive disease. Stroke 13 (1982) 189-195

Cosnett, J.E., D.J. Pudifin: Embolic complications of cardiomyopathy. Br. Heart J. 26 (1964) 544-548

Coulshed, N., E.J. Epstein, C.S. McKendrick, R.W. Galloway, E. Walker: Systemic embolism in mitral valve disease. Br. Heart J. 32 (1970) $26-34$

Daley, R., T.W. Mattingly, C.L. Holt, E.F. Bland, P.D. White: Sy stemic arterial embolism in rheumatic heart disease. Am Heart $J$. 42 (1951) 566-581

Delius, W., H. Sebening, $N$. Weghmann, $K$. Oversohl, A. Wirtzfeld, $P$. Mathes: Klinik und Verlauf der kongestiven Kardiomyopathie ungeklärter Ätiologie. Dtsch. Med. Wochenschr. 101 (1976) $635-641$

Demakis, J.G. A. Proskey, S.H. Rahimtoola: The natural course of alcoholic cardiomyopathy. Ann. Intern. Med. 80 (1974) 293297

Dewar, H.A., D. Weightman: A study of embolism in mitral valve disease and atrial fibrillation. Br. Heart J. 49 (1983) 133-140

Easton, J.D., D.G. Sherman: Management of cerebral embolism of cardiac origin. Stroke 11 (1980) 433-442

Fairfax, A.J., C.D. Lambert, A. Leatman: Systemic embolism in chronic sinoatrial disorder. N. Engl. J. Med. 295 (1976) 190-192

Fieschi, C., A. Francia, L. Allori, C. Argentino, S. Bernardi, G. Bertazzoni, F. Carpinteri, V. Di Piero, M. Ferroluzzi, G.L. Lenz, M. Prencipe, $S$. Servi, $E$. Zanette: Mitral valve prolapse as a risk factor for TIA. Eur. Neurol. 22 (1983) 233-239

Fisher, C.M., R.D. Adams: Observations on brain embolism with special reference to the mechanism of hemorrhagic infarction. $J$. Neuropathol. Exp. Neurol. 10 (1950) 92-93

Fleming. H.A., S.M. Bailey: Mitral valve disease, system ic embolism and anticoagulants. Postgrad. Med. J. 47 (1971) 599-604

Forfar, J.C. H.C. Miller, A.D. Toft: Occult thy rotoxicosis: a correctable cause of ,idiopathic" atrial fibrillation. Am. J. Cardiol. 44 (1977) $9-12$

Friedli, B., N. Aerichide, P. Grondin, L. Campean: Thromboembolic complications of heart valve protheses. Am. Heart J. 81 (1971) $702-708$

Furlan, A.J., S.J. Cavalier, R.E. Hobbs, M.A. Weinstein, M.T. Modie: Hemorrhage and anticoagulation after nonseptic embolic brain infarction. Neurology 32 (1982) 280-282

Gahl, K., H. Schliack: Zerebrale Zirkulationsstörungen bei kardialen Erkrankungen. Akt. Neurol. 7 (1980) $31-40$

Garraway, W.M., J.P. Whisnant, L.T. Kurland, W.M. O'Fallon: Changing pattern of cerebral infarction. Stroke 10. 1945-1974. (1979) 657-663

Gautier, J.C., D. Morelot: Cardiac embolism and arterial hypertension as risk factors of cerebral infarction. In: Brain and Heart Infarct. Springer Berlin, Heidelberg, New York (1977) $201-205$

Gottstein, $K$.: Pathogenese und Risikofaktoren der zerebralen Ischämie. Akt. Neurol. 4 (1977) 65-76

Grindal, A.B., R.J. Cohen, R.F. Saul, J.R. Taylor: Cerebral infarction in young adults. Stroke 9 (1978) 39-42

Hakim, M., A.J. Furlan, R.G. Hart, D.G. Sherman, A.R. Ryder-Cook, R. Hobbs, V.T. Miller, F.R. Yatsu, J. McAnulty, R.L. Koller, J.D. Easton, M. Kaste, J.P. Mohr (Cercbral Embolism Study Group) Immediate anticoagulation of embolic stroke: a randomized trial. Stroke 14 (1983) 668-676

Harris, A.W., S.A. Levine: Cerebral embolism in mitral stenosis. Ann. Intern. Med. 15 (1941) 637-643

Harrison, M.J.G., J.R. Hampton: Neurological presentation of bacterial endocarditis. Br. Med. J. 2 (1967) 148-151

Hart, R.G., B.M. Coull, D. Hart: Early recurrent embolism associated with nonvalvular atrial fibrillation: a retrospective study. Stroke 14 (1983) 688-693

Hassan, A.H., N.W. Choi, P.H. Hsu, K.K. Maini: Prognostic factors in the survival of $1484 \mathrm{Stroke}$ cases observed for 30 to 48 months. Arch. Neurol. 35 (1978) 213-218
Jörgensen, L., A. Torvik: Ischemic cerebrovascular diseases in an autopsy series. Part 2. J. Neurol. Sci 9 (1969) 285-320

Jones, H.R., L.R. Caplan, P.C. Come, N.W. Swinton, D.J. Breslin: Cerebral emboli of paradoxical origin. Ann. Neurol. 13 (1983) 314-319

Kayser-Gatchalian, M.L.: Die konjugierte Blicklähmung, ein prognostisch relevantes Symptom beim ischämischen zerebrilen Insult. Nervenarzt 54 (1983) 420-422

Kelley, R.E., J.R. Berger, M. Alter, A.G. Kovacs: Cercbral is she$\mathrm{mia}$ and atrial fibrillation. Prospective study. Neurology 34 (1984) 1285-1291

Koller, R. L.: Recurrent embolic cerebral infarction and anticoagulation. Neurology 32 (1982) 283-285

Kröss, R., B. Knittel, G. Thurnher: Zur Frage der kardialen Verursachung von zerebrovaskulären Syndromen. Wiener klinitiche Wochenschrift 93 (1981) 395-397

Kuhn, H., R. Becker, J. Fischer, J.M. Curtius, B. Lösse. W. H'ort, F. Loogen: Untersuchungen zur Ätiologie, zum Verlauf und zur Prognose der dilatativen Kardiomyopathie (DCM). Z. Kardiol. 71 (1982) 497-508

Kuramoto, K., S. Matsushita, H. Yamanouchi: Atrial fibrillation as a cause of myocardial and cerebral infarctions. Japane ie Circulation J ournal 48 (1984) 67-74

Lichtlen, P.: Endokarditis und ihre Auswirkung auf das Zen1ralnervensystem. In: Verhandlungen der Deutschen Gesellsc haft für Neurologie, Band 3. Gänshirt, H., P. Berlit, G. Haack (Hrsg.); Springer Verlag Berlin, Heidelberg, New York, Tok yo 1935

Lillicrap, D., A. Piesowicz: Mitral stenosis and systemic emboli. Br. Med. J. 2 (1964) 1169-1171

Matsumoto, N., J.P. Whisnant, L.T. Kurland et al.: Natural history of stroke in Rochester, Minnesota, 1955 through 1969: An extension of previous study, 1945 through 1954. Stroke 4 11973) $20 \div 29$

McAllen, P.M., J. Marshall: Cerebrovascular incidents after niyocardial infarction. J. Neurol. Neurosurg. Psychiatry 40 (1977) $951-955$

Meyer, J.S., J.Z. Charney, V.M. Rivera, N.T. Methew: Cereb al embolization: prospective clinical analysis of 42 cases. Stroke 2 (1971) $541-554$

Millikan, J.A.: Atrial fibrillation and embolism. Can. Med. Assoc. J. 128 (1983) $1370-1372$

Mohr, J.P., L.R. Capaln, J.W. Melski, R.J. Goldstein, G.W. Duncan, J.P. Kistler, M.S. Pessin, H.L. Bleich: The Harvard coopenative stroke registry. Neurology 28 (1978) 754-762

Sacco, R.L., P.A. Wolf, W.B. Kannel, P.M. McNamara: Survival and recurrence following stroke. The Framingham Study. Stroke 13 (1982) 290-295

Sage, J.S., R.L. van Uitert: Risk of recurrent stroke in patients with atrial fibrillation and non-valvular heart disease. Strcke 14 (1983) $537-540$

Sindermann, $F$, W. Kuntze: Bewußtseinsverlust beim Schlag.ınfall. Arch. Psychiatr. Nervenkr. 214 (1971) 262-277

Sturgill, B.C., M.G. Netsky: Cerebral infarction by atheromatous emboli. Arch. Pathol. 76 (1963) 186-196

Torvik, A., L. Jörgensen. Thrombotic and embolic occlusion; of the carotid arteries in an autopsy series. Part. 2. Cerebral :esions and clinical course. J. Neurol. Sci. 3 (1966) 410-432

Van-Horn, G., A. Hawes: Global aphasia without hemiparesis: a sign of embolic encephalopathy. Neurology. 32 (1982) 4(13-406

Vost, A., D.A. Wolochow, D.A. Howell: Incidence of infarcts of the brain in heart disease. J. Pathol. Bacteriol. 88 (1964) $463-470$

Weisberg, L.A.: Nonseptic cardiogenic cerebral embolic stroke: Clinical-CT correlations. Neurology 35 (1985) 896-899

Wells, C.E.: Cerebral embolism. Arch. Neurol. Psychiatry 81 (1959) $667-677$

Whisnant, J.P., J.P. Fitzgibbons, J.P. L.T. Kurland: Natural history of stroke in Rochester, Minnesota, 1945 through 1954. Siroke 2 (1971) $11-22$

Wolf, P.A., T.R. Dawber, K.E. Thomas, W.B. Kannel: Epideniologic assessment of chronic atrial fibrillation and risk of strcke: The Framingham Study. Neurology 28 (1978) 973-977

Wolf, P.A., W.B. Kannel, D.L. McGee, S.L. Meeks, N.E. Bharucha, P.M. McNamura: Duration of atrial fibrillation and imminence of stroke: The Framingham Study. Stroke 14 (1983) 664667 
Zang Xu: Cerebral embolism. Pathologic analysis of 16 autopsies. Chinese Med. J. 92 (3) (1979) 199-210
Zatuchni, J., H. Shenkin, H. Fisher: Arteriography in so-called cerebral embolism. Am. J. Med. Sci. 4 (1961) 468-473

Priv.-Doz. Dr. med. P. Berlit, Oberarzt der Neurologischen Klinik des Klinikums Mannheim der Universität Heidelberg, Theodor-Kutzer-Ufer, D.6800 Mannheim 1 